\title{
Development of a portable DAQ-based Electroencephalogram System
}

\author{
Saeed Mohsen \\ Ain Shams \\ University \\ Cairo, Egypt
}

\author{
Abdelhalim Zekry \\ Ain Shams \\ University \\ Cairo, Egypt
}

\author{
Mohamed \\ Abouela \\ Ain Shams \\ University \\ Cairo, Egypt
}

\author{
Ahmed Elshazly \\ ElGezeera Academy \\ Cairo, Egypt
}

\begin{abstract}
This paper presents a virtual electronic system for measuring the EEG signals. The system consists of electrodes, an instrumentation amplifier, filters and a DAQ card with LabVIEW application on a personal computer. The system is developed for displaying, measuring, analyzing and recording the EEG signals. The system is practically implemented with success where the experimental results are verified with simulation results.
\end{abstract}

\section{Keywords}

Electroencephalogram (EEG), Data Acquisition (DAQ), Laboratory Virtual Instruments Engineering Workbench (LabVIEW).

\section{INTRODUCTION}

The EEG is important in the medical field. The EEG is used to record the brain activities that are used in diagnoses strokes. Recently, the advancement in technology of the brain signals enabled the control of equipment that could help the humans in their daily life such as wheelchair and robots. Now, mindwave and emotive epock are used in EEG systems. Berger is the inventor of electroencephalography and discoverer of the alpha wave rhythm. Many EEG acquisition systems were developed [1-5]. Most of these systems are used to monitor the patient suffering from clinical disorder related to the brain [6-8]. This requires that the patient to be in health care center under the constant supervision for diagnoses $[9$, 10]. Hence, the EEG system is developed in order to be portable. The portability is in the first step is based utilizing a data acquisition card DAQ and laptop. The EEG system is a low cost system, since LabVIEW plotter application is developed the EEG system. So, the main target is to design and implement a light weight EEG system with three electrodes. These electrodes are used to sense the signals on human brain which are produced by neurons. The main problems with the brain electrical signals are that they are very small $(10 \mathrm{u} \mathrm{V}-100 \mathrm{u} \mathrm{V})$ [11]. So, they have to be amplified with special amplifiers. Such amplifiers are called instrumentation amplifiers. These amplifiers are characterized by high gain and common mode rejection ratio in addition to high input impedance. With these specifications, human brain signals can be amplified to get the EEG signal. The filter circuits are also required to clean the contamination and artifacts in EEG signal. The EEG system is implemented based on our design with DAQ card and computer. The paper is organized as follows: in section 2, the EEG system design will be presented. Then in section 3, the practical implementation of EEG circuits will be introduced. In section 4, the LabVIEW software implementation will be described in detail. Section 5 presents the experimental results and the conclusion is described in section 6 .

\section{DESIGNING OF AN EEG SYSTEM}

The block diagram of the EEG system is shown in Figure 1.It consists of electrodes to access the brain signal, an instrumentation amplifier to amplify the accessed signal, Filter to limit the bandwidth of the accessed brain signal, a gain block to achieve proper signal strength for displaying and recording, A DAQ card to acquire the signal for a laptop or a pc. In the next subsections, these components will be described in detail.

\begin{tabular}{|l|l|}
\hline Electrode 1 \\
\hline Electrode 2 \\
\begin{tabular}{|c|c|}
\hline Electrode 3 \\
\begin{tabular}{|c|c|} 
Computer \\
Amplifier \\
(LabVIEW Application)
\end{tabular}
\end{tabular}$\rightarrow \begin{array}{c}\text { DAQ Card } \\
\text { (A/D) }\end{array}$ \\
\hline
\end{tabular}

Fig 1: Block diagram of EEG system.

\subsection{EEG Electrodes}

In order to measure and record the potential from the body, it is necessary to provide interface between body and potential measuring electronics apparatus. The EEG signal from the brain is taken using electrodes placed on the scalp region of head. The electrodes used for this acquisition are made up of $\mathrm{Ag}-\mathrm{AgCl}[12]$.

\subsection{Instrumentation Amplifier}

The instrumentation amplifier circuit is shown in Figure 2. After accessing the brain signal by the appropriate electrodes, AD620 has been used as an instrumentation amplifier. The amplifier chip AD620 has Gain reaching to 10000 and its CMMR is $100 \mathrm{~dB}$. The gain of this amplifier can be calculated by using the formula: $\mathrm{G}=\left(1+\left(49.4 \mathrm{~K} \Omega / \mathrm{R}_{3}\right)\right)$. Hence, the gain can be adjusted by changing $R_{3}$. Here $R_{3}$ is selected to be 470 $\Omega$ so, to achieve gain nearly equals 107 .

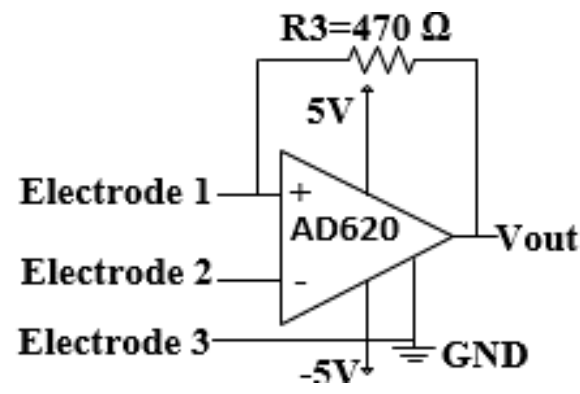

Fig 2: Instrumentation Amplifier. 


\subsection{Band pass filter \& Gain stage}

The bandpass filter circuit is depicted in Figure 3. It is a simple one stage high pass C-R filter followed by a lossy integrator acting as a low pass filter and gain block. High pass cut-off frequency is roughly $0.5 \mathrm{~Hz}$ and low pass filter cut-off frequency is nearly $34 \mathrm{~Hz}[13,14]$. The frequency response of designed circuit is shown in Figure 4. The midband gain of 100 and the $3-\mathrm{dB}$ cut off points of $0.35 \mathrm{~Hz}$ and $24 \mathrm{~Hz}$.

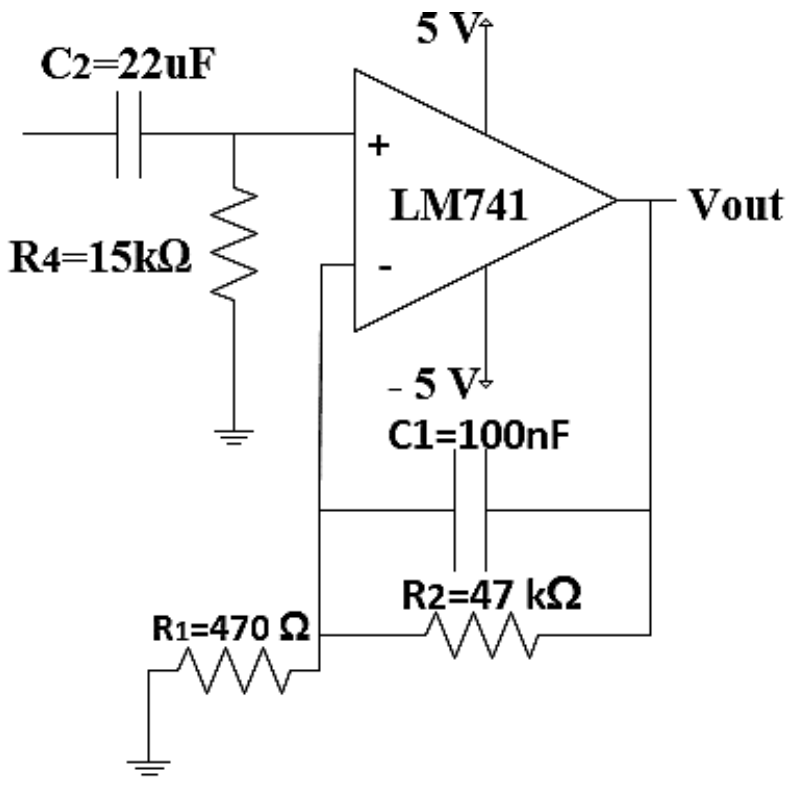

Fig 3: Band pass filter and gain stage.

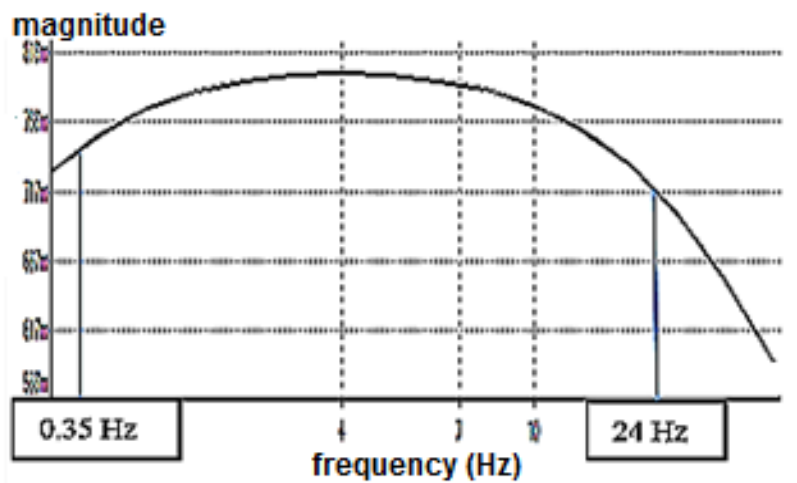

Fig 4: Frequency response of designed circuit.

\subsection{Power Management Circuit}

The analog electronic part of the interface circuit must be supplied with the proper power supply. May be the best power supply to avoid interference with the public supply is to use chemical rechargeable batteries. In addition, this system is aimed to develop a portable instrumentation. The power supply range for operational amplifiers is selected to be in low voltage range which can be supplied by small batteries. So, this circuit is designed to work on the voltage range of above three volts and under $5 \mathrm{~V}$. Also, there is a need to supply constant power supply to the operational amplifiers since the battery voltage is degraded with continuous usage. Hence, a constant $\pm 5 \mathrm{~V}$ power is extracted from a $9 \mathrm{~V}$ battery using $5 \mathrm{Vvoltage}$ regulators. Two $9 \mathrm{~V}$ rechargeable Batteries are used to supply power to the circuit by connecting them in series as shown in Figure 5. The positive terminal of one battery and negative terminal of another battery are connected together to form a zero voltage ground terminal. The capacitors are used on either side of the regulator to supply clean DC power to the operational amplifiers. The $9 \mathrm{~V}$ power supplies are regulated using UA7805 for $5 \mathrm{~V}$ and UA7905 for $-5 \mathrm{~V}$ to provide a constant power supply voltage to the operational amplifiers. The whole power supply circuit is shown in Figure 5.

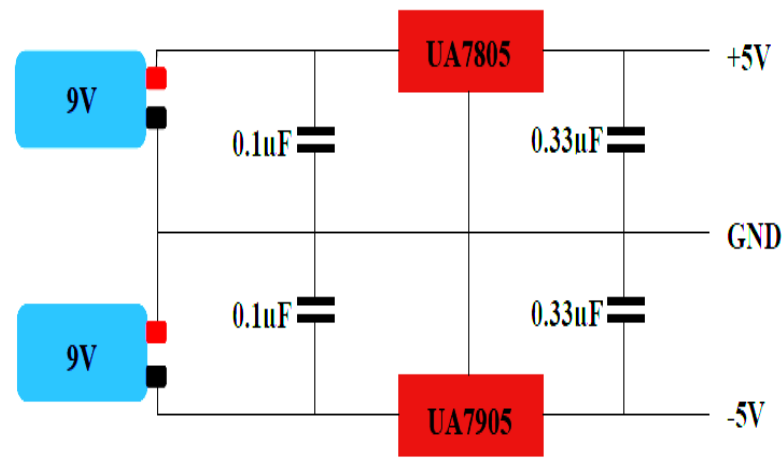

Fig 5: Power supply circuit schematic diagram.

\subsection{Data Acquisition}

The function of data acquisition is to acquire an electrical or physical phenomenon such as voltage. Also, Data Acquisition is the process of analyzing and saving the data. Data Acquisition card (DAQ) acts as connection between the computer and the outside world. It functions as a device that digitalizes incoming analog signals that are interpreted by the computer. There are different types for DAQ cards. The 6008 DAQ card is satisfactory for purposes while cost effective. This DAQ card is provided by National Instruments and has some properties as small size, compatible with LabVIEW, eight analog inputs, two analog outputs, 12 digital $\mathrm{I} / \mathrm{O}$ and USB connection. Also, the 6008 DAQ card has 12 bit resolution.

\section{EEG CIRCUIT IMPLEMENTATION}

After simulating the circuit is verified by bread board until satisfactory results were achieved. The final EEG circuit is laid out on a printed circuit board by using ARES software. The circuit lay out is shown in Figure 6 while Figure 7 shows The EEG circuit is construction on PCB. The power supply circuit is fully constructed as shown in Figure 8.

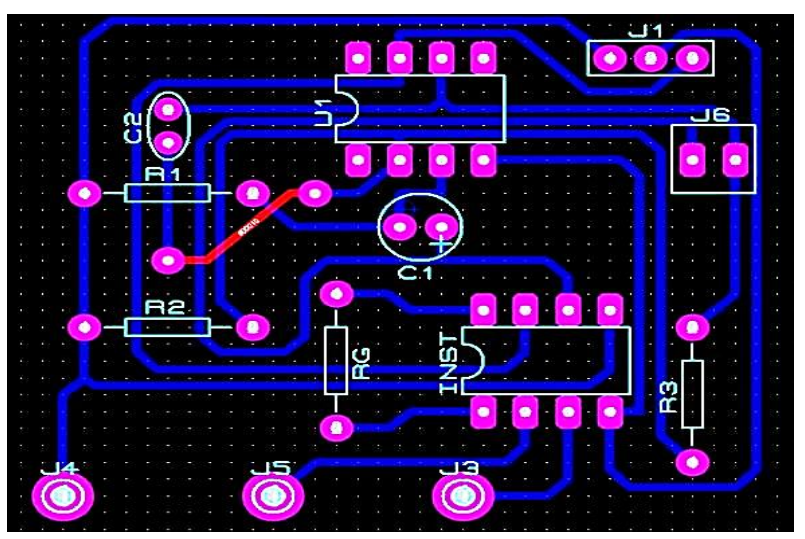

Fig 6: Final design by using ARES Software.

Printing the circuit to a PCB helps reduce some noise in the EEG system and allow for a much smaller product as shown in Figure 6. Only four resistors, two capacitors, two integrated circuits and three electrodes are used in our EEG circuit implementation. 


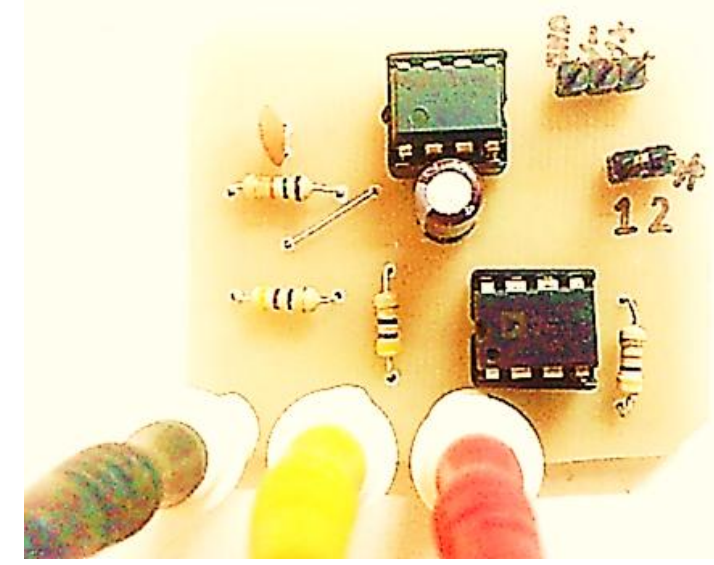

Fig 7: Final PCB construction of EEG circuit.

\section{LABVIEW SOFTWARE PLOTTER DEVELOPMENT}

In this section, the developed plotter application on the LabVIEW will be described. LabVIEW is graphical programming language which uses blocks instead of lines of text to create programs. LabVIEW is produced by National instruments. The LabVIEW is installed on the windows system which operates on a computer. The plotter application is developed on LabVIEW whose block diagram is depicted in Figure 9. It is developed for acquiring, controlling, measuring, analyzing, processing and saving the EEG signals . The DAQ Assistant block is used to read an analog input signal from the DAQ card that receives the output of the EEG circuit. The Configuration of the DAQ Assistant involves setting the expected input range to the DAQ device and sampling rate. The DAQ Assistant express block includes options for setting which hardware channel to use and selecting the acquisition mode of the device. There are four blocks which are developed for displaying the signals.

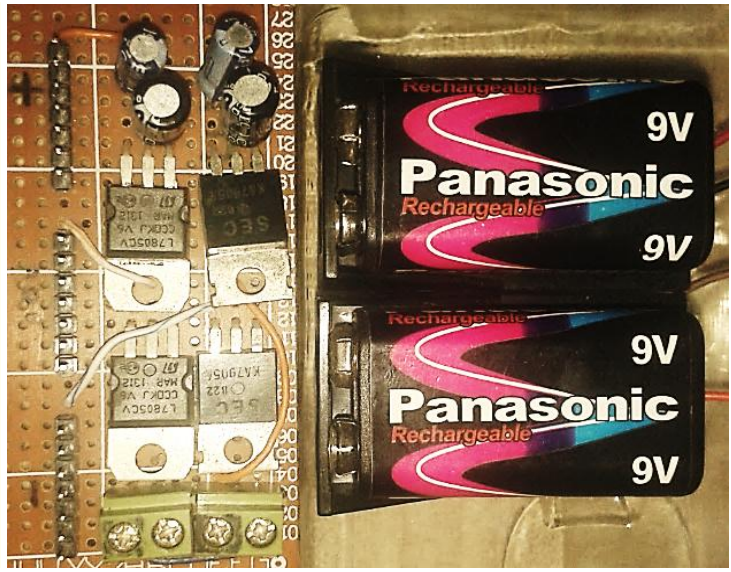

Fig 8: Construction of power supply circuit.

The DAQ Assistant block wired to one of them as Waveform graph to observe the EEG signal in time domain. The vertical pointer slide control placed and wired it to the y terminal of multiply function to adjust the gain. The maximum range of the vertical pointer slide control is five for the gain changing. Also, a filter block is created to smooth the incoming data. The Write to Measurement file block is used to save the data in a file. The configuration of the Write to Measurement expresses the file name text box that contains the full path to the output file. LabVIEW saves data with up to six digits of precision in .lv $\mathrm{m}$ file. The FFT blocks are developed to transform the data in the frequency domain because it is easier to analyze the EEG signal originally in time domain, in the frequency domain. The presence of specific frequencies is an indicator of different brain states such as resting and sleeping [11]. The graphical user interface panel based on LabVIEW plotter application developed in Figure 9 is depicted in Figure 10 where it shows the virtual instrument controls and displays.

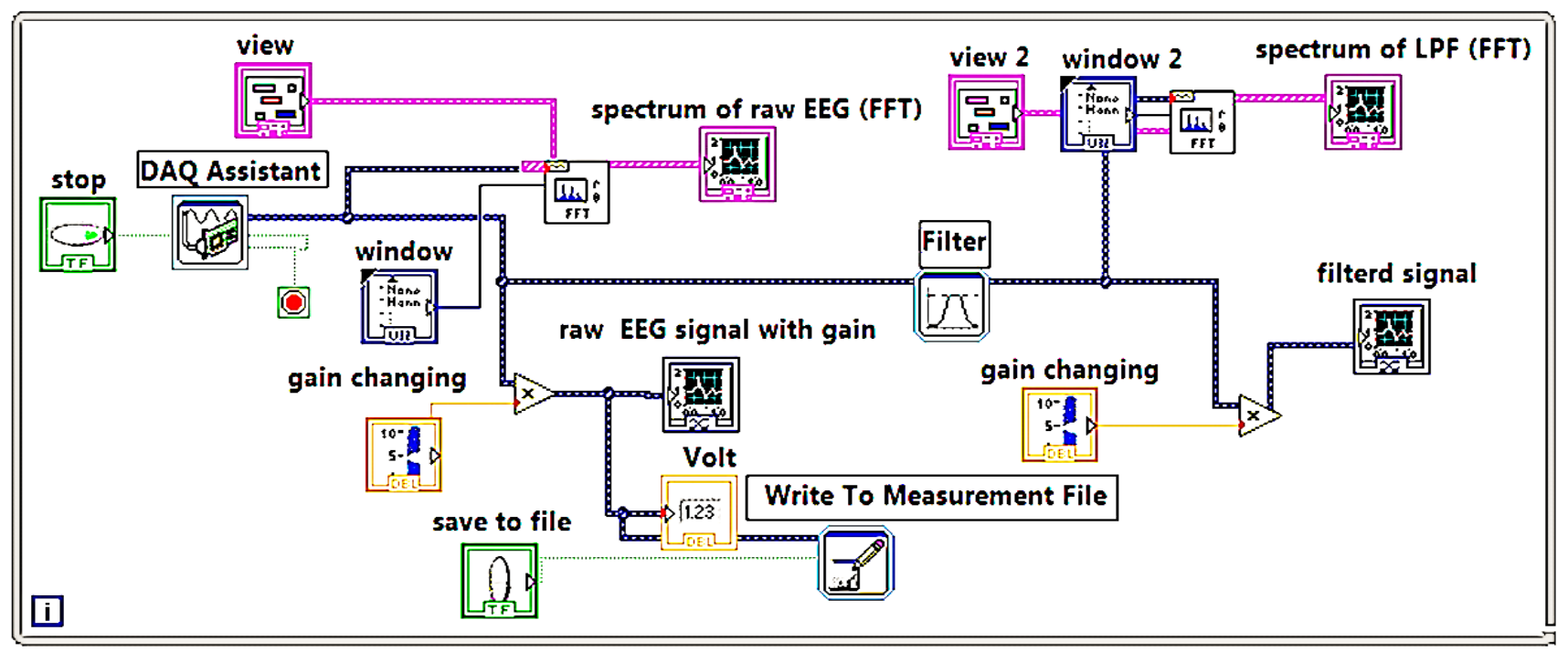

Fig 9: LabVIEW block diagram.

This panel consists of two buttons. One button is (stop (F)) that is used to stop the running and displays the final status on the screen. The other button is (save to file) that is used to save the acquired data from the connected device. There are two vertical pointer slides used to change the signal gain.
Also, two waveform graphs are created to display the output signals in time domain on the front panel. Moreover, two FFT spectrum graphs are created to display the frequency spectrum of the brain signals. 


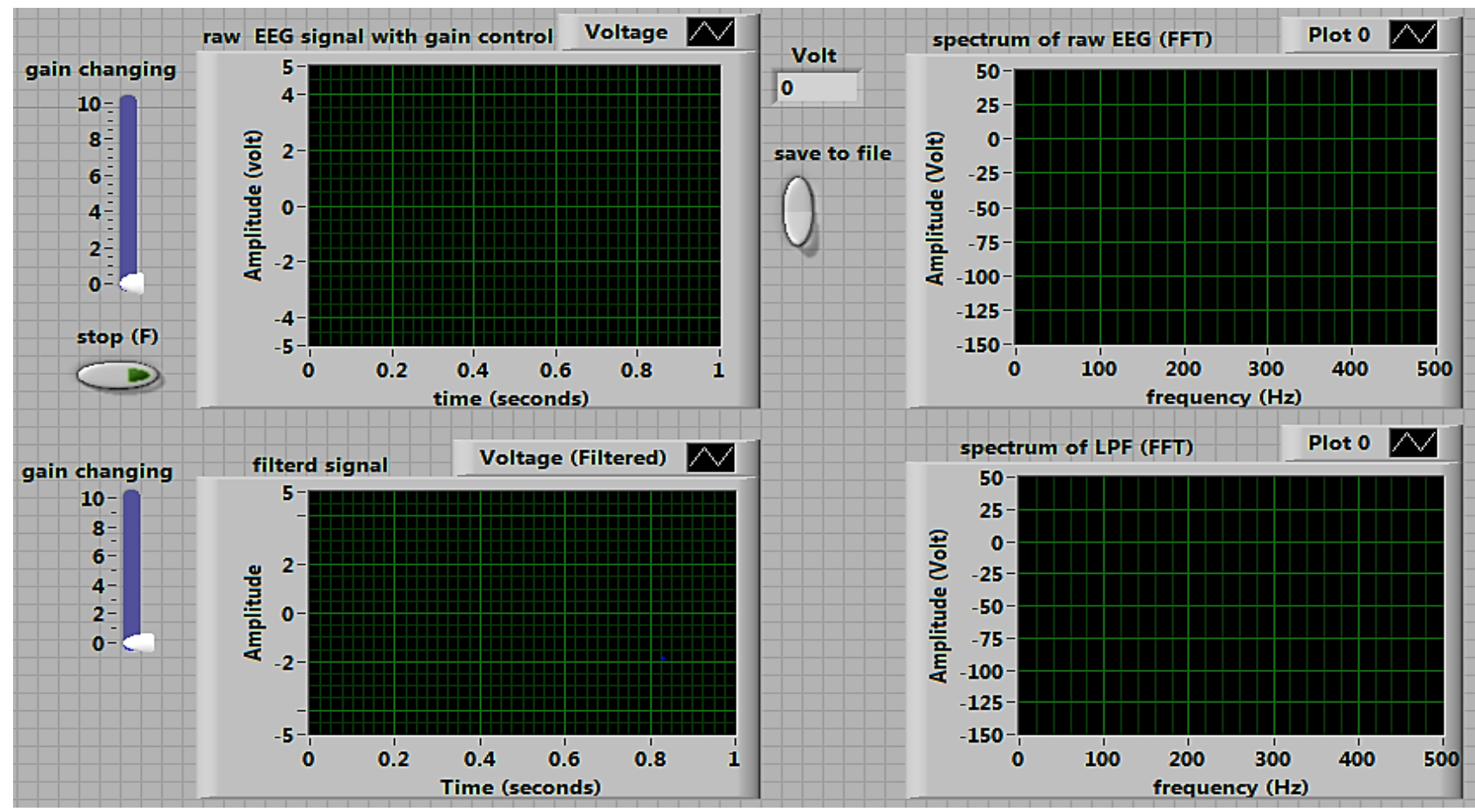

Fig 10: EEG plotter control and display panel on a Computer.

\section{EXPERIMENTAL RESULTS}

Now to demonstrate the ordinary operation of our virtual instrument based EEG system, it is used to display and process the acquired EEG signal from a normal person. Therefore, figure 11 shows typical recording of EEG raw signals for 1 second at sampling rate $1 \mathrm{k}$ samples.

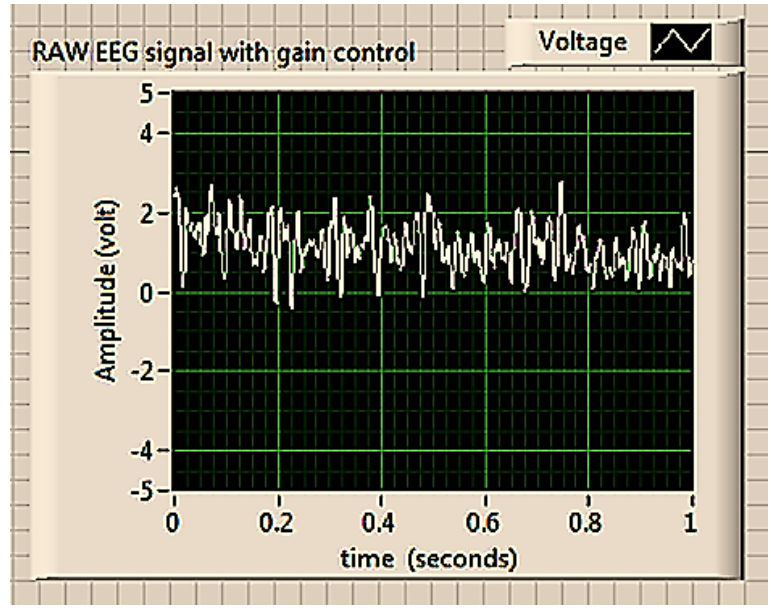

Fig 11: EEG signal for 1 second at $1 \mathrm{k}$ samples.

Figure 12 depicts the fast Fourier transform of the EEG wave from shown in Figure 11. It is clear from the figure that the amplitude of the frequency components decrease with time as observed with ordinary EEG signals.

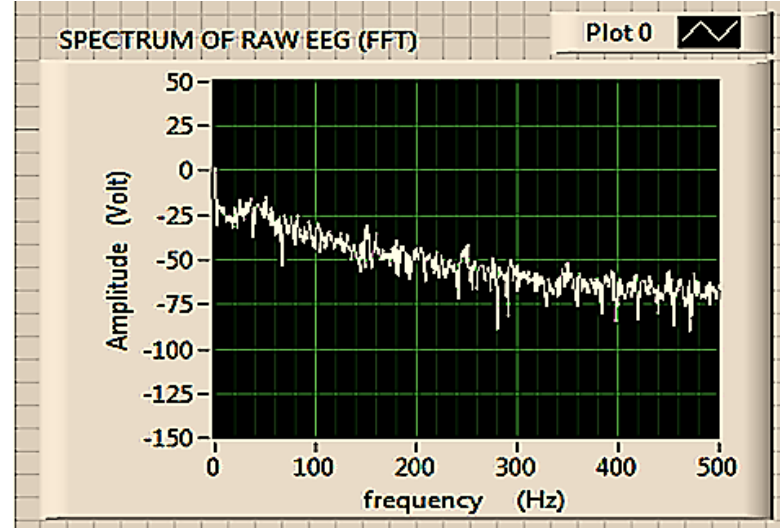

Fig 12: spectrum of raw EEG signals at 1k samples

In order to see the effect of the sampling on the EEG signal acquisition process, figure 13 shows the recording of EEG signals for 0.5 second at sampling rate of $2 \mathrm{k}$ samples.

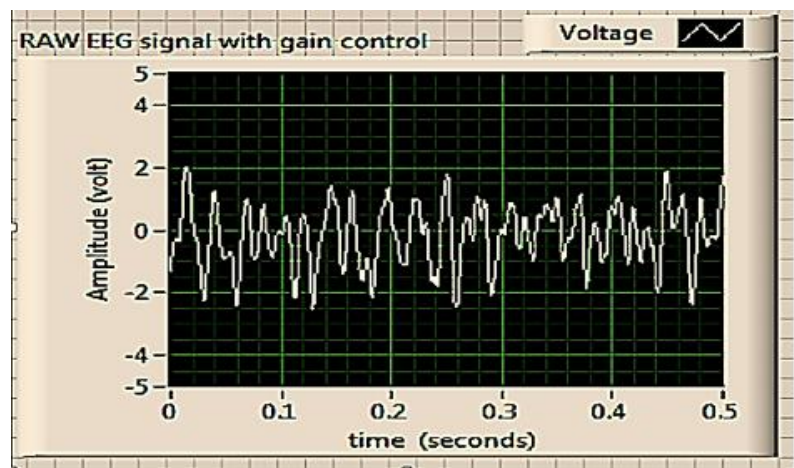

Fig 13: EEG signal for 0.5 second at $2 \mathrm{k}$ samples.

The frequency spectrum of the EEG signal sampled at the double rate seems smoother than that at lower sampling rate. As shown in Figure 14. But, they almost have the same shape. This is because the lower sampling rate is much higher than 
the Nyquist sampling rate which is double the highest frequency contained in the EEG signal. The highest frequency of the EEG signal is limited to $34 \mathrm{~Hz}$.

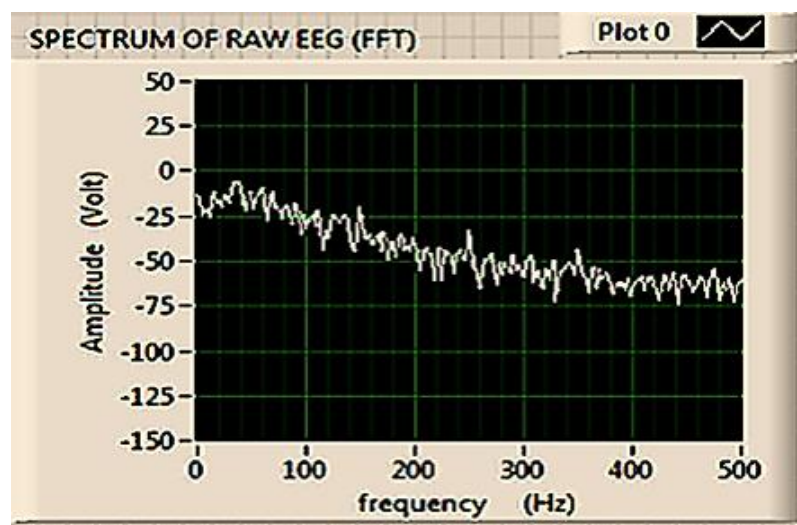

Fig 14: spectrum of raw EEG signals at 2k samples.

In order to see the effect of the digital filter on the EEG signal, figure 15 shows the filtered EEG signals for 0.5 second at sampling rate $2 \mathrm{k}$ samples. The digital filter used is a third order FIR low pass filter and is characterized by cut off frequency of $34 \mathrm{~Hz}$. The magnitude response of the digital filter is shown in Figure 16. It is clear that the filter has great effect on the shape of the waveform where the signal approaches the sinusoidal waveform.

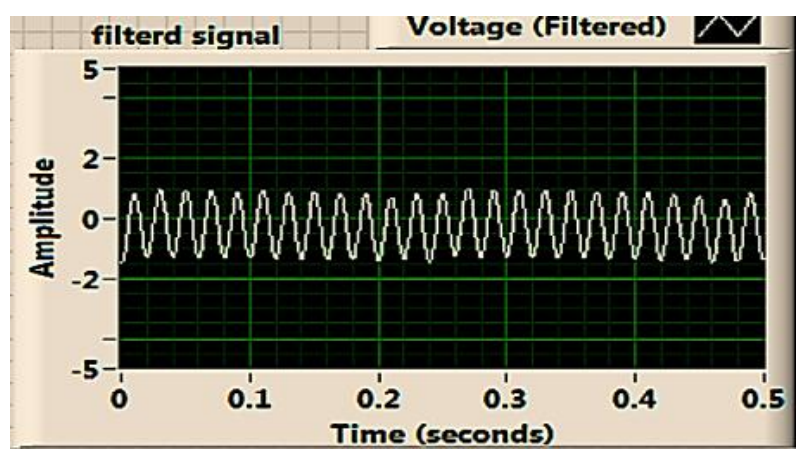

Fig 15: filtered EEG signals for 0.5 second at sampling rate $2 \mathrm{k}$ samples.

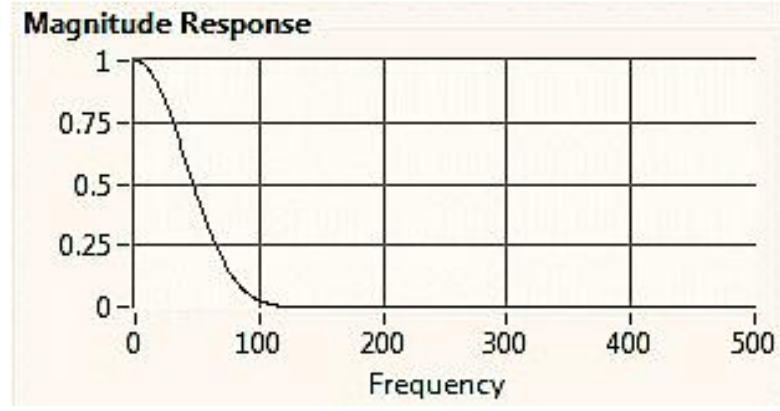

Fig 16: The magnitude response of the Low pass FIR digital filter.

Finally, Figure 17 shows a comparison between these results and the results given in referece [15]. The two signals resembles each other to great extent in spit of belonging to different persons.

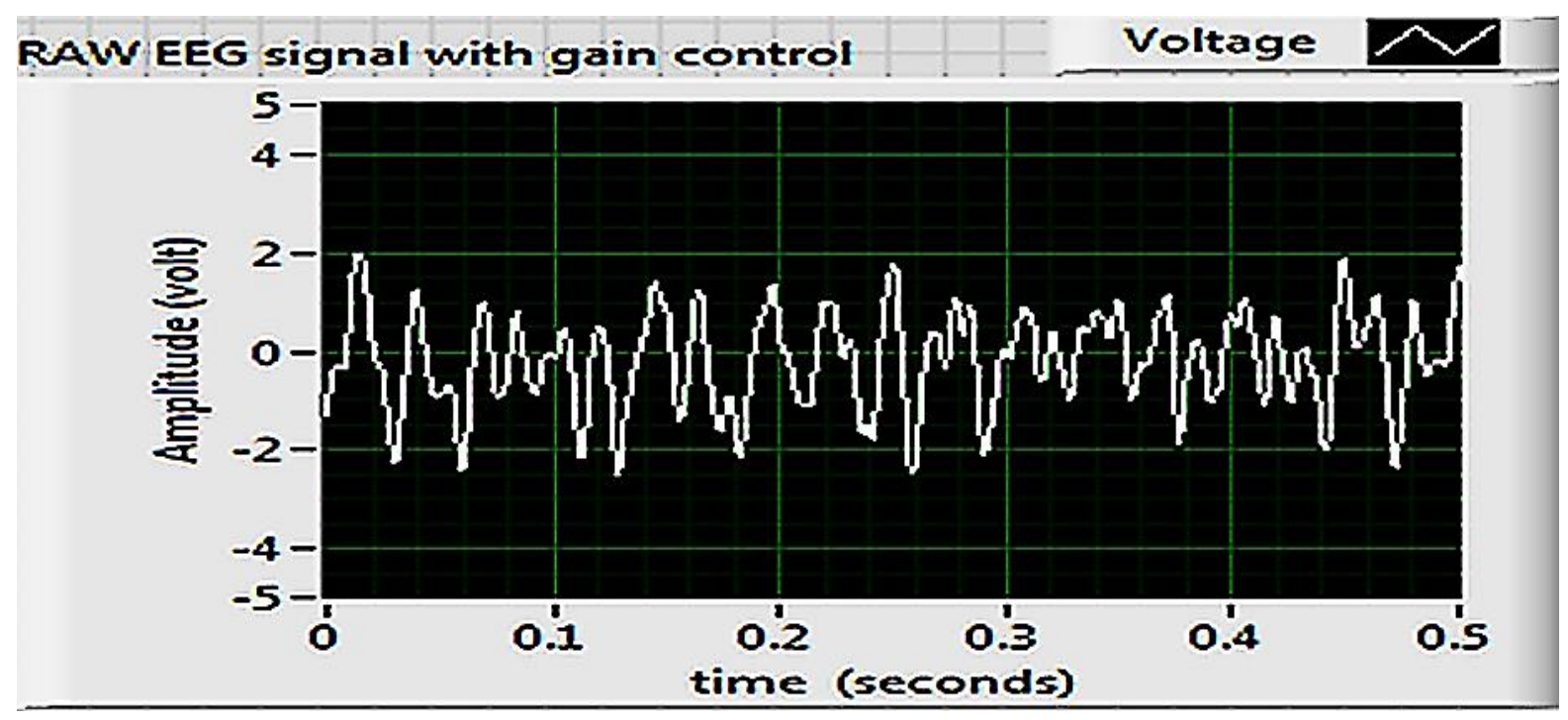

(a) 


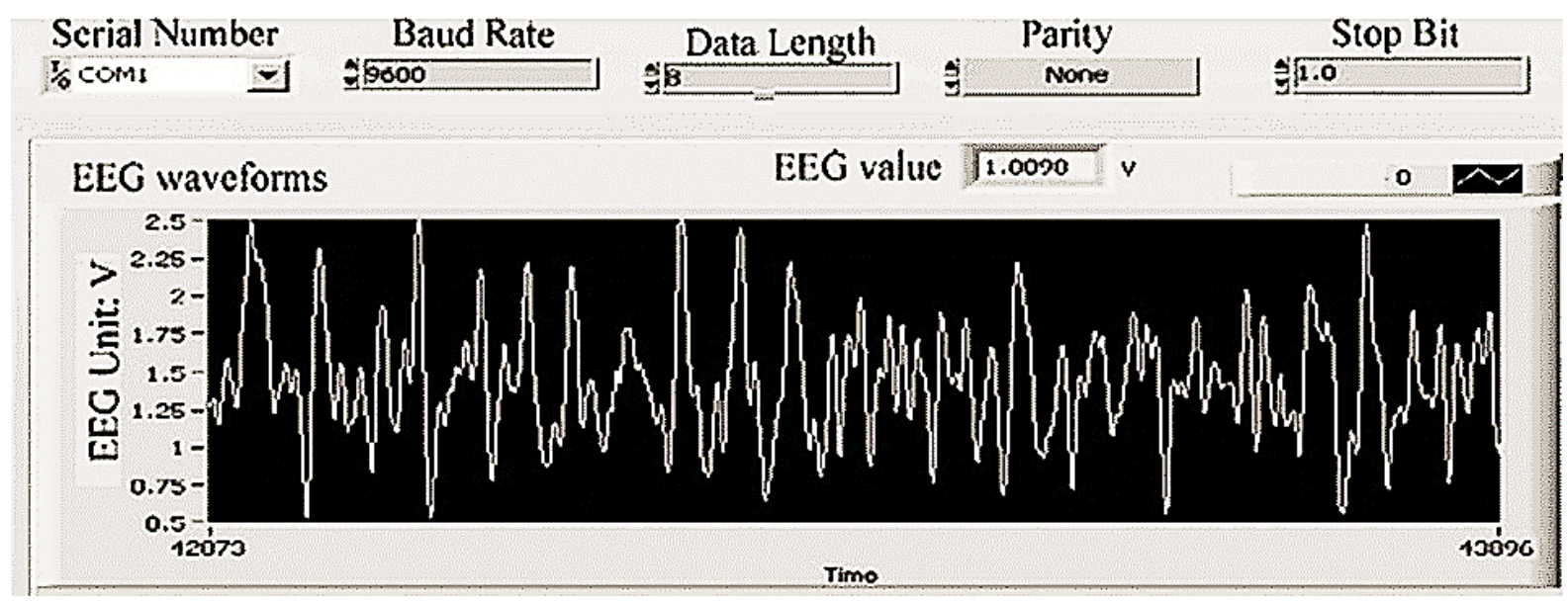

(b)

Fig 17: Comparison between two EEG rhythms: (a) recorded rhythm, (b) published rhythm in paper [15].

\section{CONCLUSIONS \& FUTURE WORK}

This paper introduces a full operational and low cost electronic system for acquiring and processing the EEG signals. The developed system consists of an analog circuit that interfaces a data acquisition card connected to Lap Top or PC. The software required to operate the setup is developed using LabVIEW in time domain and frequency domain. The developed setup is tested and proved to work properly. In this way one can develop an economical reliable test equipment for biomedical applications which can displace the very expensive instruments. Also, one can extend the system for multiple probe operation.

\section{REFERENCES}

[1] N. Verma et al, "A Micro-Power EEG Acquisition SoC with Integrated Feature Extraction Processor for a Chronic Seizure Detection System", IEEE Journal of Solid-State Circuits, Vol. 45, No. 4, pp. 804-816, April 2010.

[2] R. Dilmaghani et al, "Design and Implementation of a Wireless Multi-Channel EEG Recording", in IEEE, IET International Symposium on Communication Systems, Networks and Digital Signal Processing, Newcastle, UK, pp. 741-746, 2010.

[3] X. Chen and J. Wang, "Design and Implementation of A Wearable, Wireless EEG Recording System", in 5th International Conference on Bioinformatics and Biomedical Engineering, Wuhan, CN, pp. 1-4, 2011.

[4] D.-G. Kim, K.-S. Hong, and K.-W. Chung, "Implementation of Portable Multi-Channel EEG and Head Motion Signal Acquisition System", in 8th International Conference on Computing and Network Technology, Gueongju, KR, pp. 370-375, 2012.

[5] J.-S. Lin and S.-M. Huang, "An FPGA-Based BrainComputer Interface for Wireless Electric Wheelchairs", Applied Mechanics and Materials, Vol. 284-287, pp. 1616-1621, 2013.

[6] Drakulic, B.S. Berry, S.J. Sterman, M.B., "A portable EEG recording system", Engineering in Medicine and Biology Society, 1989. Images of the Twenty-First Century., IEEE Proc. of the Annual International Conference of the IEEE Engineering, Vol. 5, pp.13951396, Nov 1989.
[7] Filipe, S. Charvet, G. Foerster, M. Porcherot, J. Beche, J.F. Bonnet, S. Audebert, P. Regis, G. Zongo, B. Robinet, S. Condemine, C.Mestais, C.Guillemaud, R., "A wireless multichannel EEG recording platform, "Engineering in Medicine and Biology Society,EMBC, IEEE 2011 Annual International Conference, pp.63196322, 2011

[8] Saadi, H. Ferroukhi, M. Attari, M., "Development of wireless high immunity EEG recording system" ,Electronic Devices, Systems and Applications (ICEDSA), 2011 International Conference, pp.120, 124, 25-27,April 2011.

[9] Junwei Duan, Changhao Chen, Sio Hang Pun, Feng Wan, Peng Un Mak, Pui In Mak, Mang I Vai, Yong Hu, "A Wearable Wireless General Purpose Bio-signal Acquisition Prototype System for Home Healthcare, "Biomedical Engineering and Biotechnology (iCBEB), 2012 International Conference, pp.1176, 1179, 28-30 ,May 2012.

[10] Nassir, A. Barnea, O., "Wireless body-area network for detection of sleep disorders", Electrical \& Electronics Engineers in Israel (IEEEI), 2012 IEEE $27^{\text {th }}$ Convention, pp.1-5, Nov 2012.

[11] RAKESH KUMAR,"BIO-MEDICAL SIGNAL PROCESSING", First edition. S.K. KATARIA \&SONS, 2009.

[12] John G Webster, "Medical Instrumentation: Application and Design, $3^{\text {rd }}$ ed", New York: John Wiley \&Sons Inc., 1998.

[13] R. Dilmaghani, M. Ghavami, K. Cumar, A Dualeh, S. Gomes Da Sousa, R. Salleh Mohd, M. Sekanderzada, H. Koke, "Design and Implementation aWireless Multi channel EEG Recording", London, pp.741-746, 2010.

[14] David Wang, "Using my DAQ and LabVIEW to Develop a Single-Channel EEG for a Multi-Modality Epileptic Seizure Detection Platform", In 39th Annual Northeast Bioengineering Conference, 2013.

[15] Yang Minglng, Ai Qingsong and Liu Quan,"Design of a High- Performance EEG Acquisition System for Unshielded Environment", in IEEE, pp.202-206, china, 2012. 